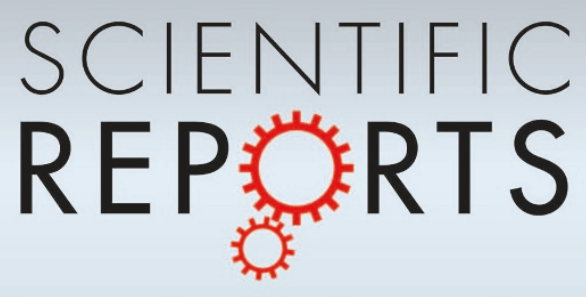

OPEN

SUBJECT AREAS:

TERAHERTZ OPTICS

OPTICAL PROPERTIES AND

DEVICES

Received

26 August 2014

Accepted

20 November 2014

Published

10 December 2014

Correspondence and requests for materials should be addressed to

Q.Y.W. (qywen@ vestc.edu.cn)

\section{Graphene based All-Optical Spatial Terahertz Modulator}

\author{
Qi-Ye Wen', Wei Tian', Qi Mao', Zhi Chen², Wei-Wei Liu', Qing-Hui Yang', Matthew Sanderson ${ }^{4}$ \\ \& Huai-Wu Zhang'
}

'State Key Laboratory of Electronic Films and Integrated Devices, University of Electronic Science and Technology of China, Chengdu, 610054, P.R.China, ${ }^{2}$ National Key Laboratory of Science and Technology of Communication. University of Electronic Science and Technology of China, Chengdu, 610054, P.R.China, ${ }^{3}$ Institute of Modern Optics, Nankai University, Key Laboratory of Optical Information Science and Technology, Ministry of Education, Tianjin, 300071, P.R. China, ${ }^{4}$ School of Physics, University of Wollongong, NSW 2522, Australia.

We demonstrate an all-optical terahertz modulator based on single-layer graphene on germanium (GOG), which can be driven by a $1.55 \mu \mathrm{m} \mathrm{CW}$ laser with a low-level photodoping power. Both the static and dynamic $\mathrm{THz}$ transmission modulation experiments were carried out. A spectrally wide-band modulation of the $\mathrm{THz}$ transmission is obtained in a frequency range from 0.25 to $1 \mathrm{THz}$, and a modulation depth of $94 \%$ can be achieved if proper pump power is applied. The modulation speed of the modulator was measured to be $\sim 200 \mathrm{KHz}$ using a $340 \mathrm{GHz}$ carrier. A theoretical model is proposed for the modulator and the calculation results indicate that the enhanced $\mathrm{THz}$ modulation is mainly due to the third order nonlinear effect in the optical conductivity of the graphene monolayer.

$\mathrm{T}$ erahertz (THz) technology offers a variety of applications including spectroscopy, imaging, and communications. Much effort has been devoted to developing $\mathrm{THz}$ sources and detectors, which has promoted $\mathrm{THz}$ research into one of the most rapidly growing fields ${ }^{1}$. However, substantive progression of $\mathrm{THz}$ applications also depends on the realization of active components for wave manipulation and modulation. One of the key components mostly desired for advanced THz system is a spatial light modulator that actively controls the spatial transmission (reflection) of an incident $\mathrm{THz}$ wave ${ }^{2}$. This kind of device enables one to encode information on $\mathrm{THz}$ waves thus provides potential applications in imaging, telecommunication, beam shaping, etc. Demonstrations of $\mathrm{THz}$ modulators have been reported based on $2 \mathrm{DEG}^{3,4}$, metamaterials ${ }^{2,4,5}$, superconductors ${ }^{6,7}$, and phasetransition materials ${ }^{8}$. An alternative means of controlling and modulating $\mathrm{THz}$ wave is based on optically induced carriers in semiconductors ${ }^{9,10}$. Very recently, an optical driven spatial THz modulator (STM), based on a plain silicon wafer, has been proposed to realize photo-designed $\mathrm{THz}$ devices ${ }^{11}$ or reconfigurable quasi-optical $\mathrm{THz}$ components ${ }^{12}$. The capability of these devices to manipulate $\mathrm{THz}$ wave front also enables one to generate special intensity patterns and vortex $\mathrm{THz}$ beams ${ }^{13}$. Unfortunately, silicon wafers exhibits a weak modulation depth of $19.9 \%$ and a limit of modulation speed less than $100 \mathrm{KHz}$ even when a large laser fluence is applied, which greatly limits the achievable tunability and versatility ${ }^{13,14}$. Therefore, optical STM with large modulation depth under moderate laser power is highly desired in producing efficient and high speed $\mathrm{THz}$ devices.

Graphene, a single layer of carbon atom with honeycomb structure, has proven useful for $\mathrm{THz}$ and optical modulation due to its unique band structure and extremely high carrier mobility ${ }^{15-19}$. With graphene, light modulators were realized with desirable features such as broadband or high-speed ${ }^{20,21}$. Recently, a graphenebased $\mathrm{THz}$ modulator was demonstrated by electrically tuning the density of states available for intraband transitions ${ }^{15,19}$. However, its modulation depth and speed are limited to $15 \%$ and $20 \mathrm{KHz}$ by the electrical device prototype. Striking progresses were made by Rahm et $\mathrm{al}^{22}$. They observed an enhanced $\mathrm{THz}$ modulation in a graphene/silicon structure over a wide frequency band. When a pulsed femtosecond laser with wavelength of $808 \mathrm{~nm}$ is incident upon the graphene/silicon, a great number of free carrier are produced in silicon and these photo-generated carries diffuse into the graphene layer, giving rise to a stronger change of conductivity than would occur in pure Si since graphene has higher carrier mobility. This phenomenon results in a THz modulation with a depth of $99 \%$ in the frequency range from 0.2 to $2 \mathrm{THz}$. However, experiments performed so far were primarily focused on the static properties and the modulation depth and speed have not been systematically studied. Furthermore, Si modulators are not efficient at wavelengths of $1.3-1.55 \mu \mathrm{m}$ due to the inherent large bandgap of $\mathrm{Si}$ thus has the problem of integrating with existing optical fiber communications ${ }^{23}$. 


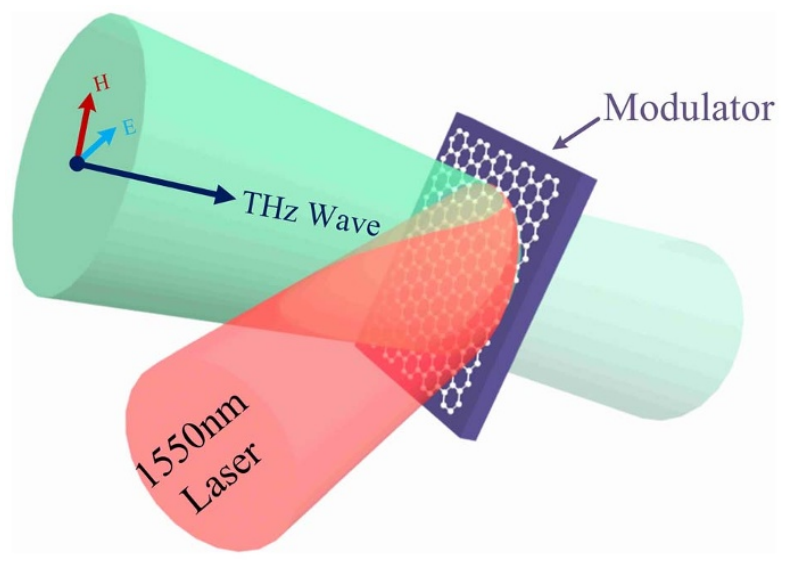

Figure $1 \mid$ Prototype and spatial configuration of the all-optical spatial terahertz modulator. The modulator consists of a single-layer graphene sheet on a germanium substrate, which is driven by a $1550 \mathrm{~nm}$ laser. The beam of the $\mathrm{THz}$ wave is completely overlapped by the laser beam.

In this work, we demonstrate a high speed, broadband and costeffective $\mathrm{THz}$ all-optical modulator. The modulator is based on graphene on germanium (GOG), which allow a pumping light with $1.3-1.55 \mu \mathrm{m}$ wavelength since Ge has a small bandgap of $0.66 \mathrm{eV}$. Another advantage of Ge compared to silicon is that Ge has higher bulk mobility for both electrons and holes, which should ideally correspond to an increase in surface mobility and ultimately an increase in the device performance such as modulation depth and speed. In the present work, both the static and dynamic THz transmission modulation experiments were carried out. A wide-band modulation of the $\mathrm{THz}$ transmission was obtained in a frequency range from 0.25 to $1 \mathrm{THz}$ under a $1550 \mathrm{~nm}$ pumping laser. A maximum modulation depth of $94 \%$ and a modulation speed of $\sim 200 \mathrm{KHz}$ were measured by using a $340 \mathrm{GHz}$ carrier. These features make GOG a viable candidate for integration with telecommunication fiber for broadband, high speed and low-cost spatial $\mathrm{THz}$ modulator.

The prototype and the spatial configuration of the GOG based STM is shown in Fig. 1. The $\mathrm{THz}$ wave is overlapped by the $1550 \mathrm{~nm}$ modulation laser beam, and both beams are incident from the graphene side. The spectral transmission of the modulator was measured by a home-made $\mathrm{THz}$ time domain spectroscopy (TDS) with and without photoexcitation. In this TDS setup, an Er: fiber laser produces $1550 \mathrm{~nm}$ pulses of $100 \mathrm{fs}$ duration with $100 \mathrm{MHz}$

(a)

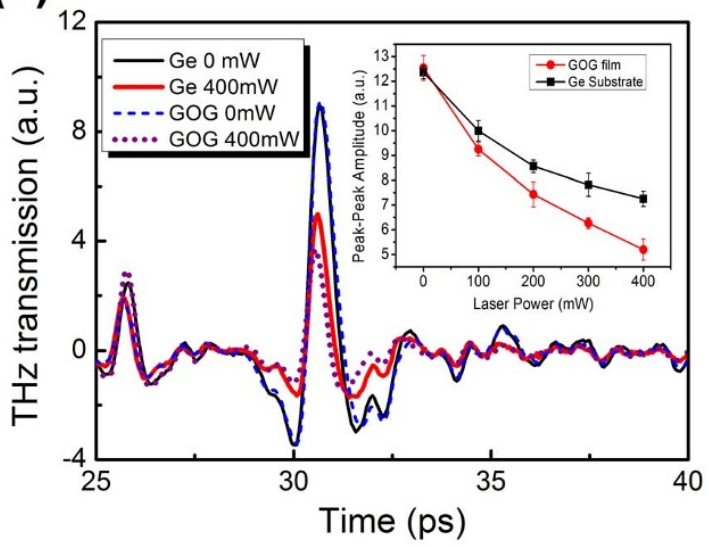

repetition rate. The source beam is split into three portions, corresponding to terahertz generation, probe, and pump beams, respectively. The pump beam has a maximum average power of $400 \mathrm{~mW}$ and a spot diameter of $5 \mathrm{~mm}$ so that it can overlap the $\mathrm{THz}$ beam $(\sim 3 \mathrm{~mm})$.

In Fig. 2, the amplitude transmittances of the $\mathrm{THz}$ modulator and the Ge substrate, with and without laser pumping, are plotted in both the time- and frequency-domain. The frequency-domain spectra are normalized to a reference spectrum measured without any sample. Upon pumping at a light fluence of $400 \mathrm{~mW}$, both GOG and pure Ge substrate show considerable transmission attenuations of $\mathrm{THz}$ wave. It is well known that free carriers can be generated in a semiconductor through photoexcitation, which forms a thin conductive layer at the surface of the sample ${ }^{10}$. The reflection and absorption by this conductive layer of a $\mathrm{THz}$ wave causes the broadband attenuation of the transmitted $\mathrm{THz}$ wave $\mathrm{e}^{10,22}$. Since the conductivity of this thin layer, $\sigma$, is proportional to the density and the mobility of the photo-induced carriers, the larger mobility of graphene gives rise to the stronger $\mathrm{THz}$ attenuation of GOG as compared to pure Ge. It is worth to point out that the modulation effect of pure Ge substrate is also quite notable since pure Ge itself has high mobility for both electrons (typically $3900 \mathrm{~cm}^{2} \mathrm{~V}^{-1} \mathrm{~s}^{-1}$ ) and holes (typically $1900 \mathrm{~cm}^{2} \mathrm{~V}^{-1} \mathrm{~s}^{-1}$ ). The peak to peak amplitude of the main pulse of the time domain spectrum is specially plotted as inset in Fig. 2 (a) as a function of the pump power. It is clear that the THz transmission for both samples decreases with the increase of the pump fluence, confirming that it is the increase of the conductivity that causes the attenuation of the transmitted $\mathrm{THz}$ wave ${ }^{22}$.

The frequency-domain spectra shown in Fig. 2(b) provide more detailed information for the broadband modulation properties. Without photoexitation, both samples show a moderate transmission of $\sim 50 \%$ of the $\mathrm{THz}$ wave due to the reflection and partial absorption from the carriers since the Ge substrate is slightly pdoped. Similar result has been reported in pure $\mathrm{Ge}$ in $\mathrm{THz}$ frequency range ${ }^{24}$. It is interesting to see that GOG exhibits a higher transmission than $\mathrm{Ge}$ at frequency beyond $0.4 \mathrm{THz}$. This phenomenon is well reproducible. The possible reason is that the graphene acts as an ultrathin anti-reflection coating to Ge, just like a parylene thin film on $\mathrm{Si}$ substrate $\mathrm{e}^{24}$, thus the transmission through GOG is slightly enhanced. Upon pumping by $1.55 \mu \mathrm{m}$ laser with a power of $400 \mathrm{~mW}$, the modulation depth averaged from $0.25 \mathrm{THz}$ to $1 \mathrm{THz}$ is $83 \%$ for GOG, while that for pure Ge is $68 \%$. The modulation depth mentioned here is defined as ( $\left.T_{\text {no pumping }}-T_{\text {pumping }}\right) / T_{\text {no pumping, }}$ where $T_{\text {no pumping }}$ and $T_{\text {pumping }}$ represent the intensity of $\mathrm{THz}$ transmission without and with light pumping respectively. The (b)

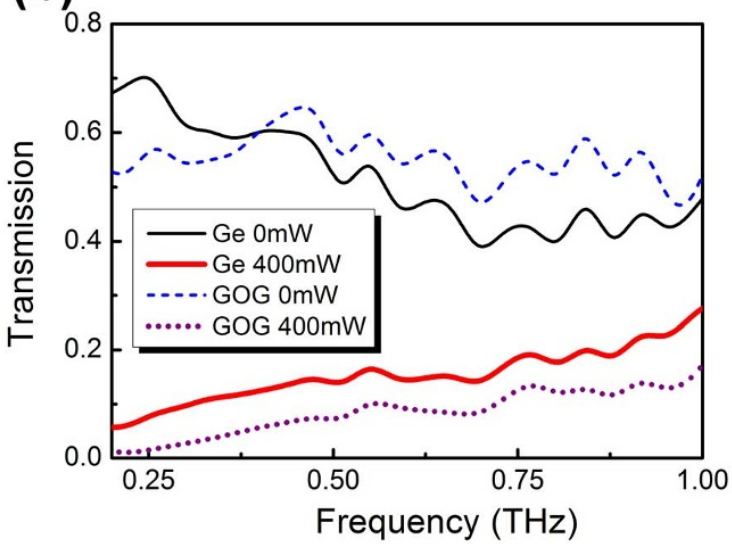

Figure $2 \mid$ Measured THz transmission from the Ge and GOG samples. The measurements were carried out on a THz-TDS system without and with photodoping at a power of $\mathrm{P}=400 \mathrm{~mW}$. (a) The time domain spectra and (b) the corresponding frequency domain spectra calculated from the time domain data. Inset in (a) plotted the peak-peak amplitude of the main THz pulse as a function of the pumping power. 


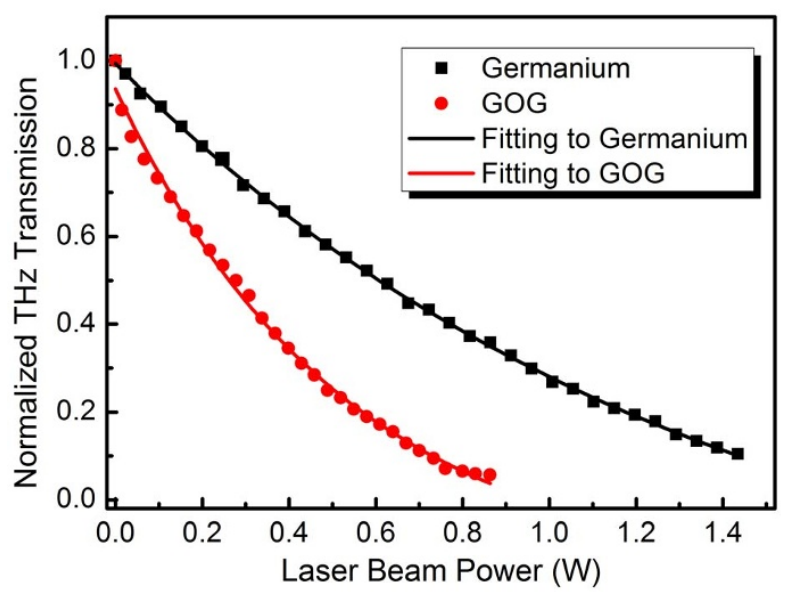

Figure $3 \mid$ Measured static modulation characteristics of the STM.

Normalized THz transmission at $340 \mathrm{GHz}$ carrier as a function of the laser beam power for the Ge and GOG. The solid lines are fitting curves to the experimental data by an exponential decay function.

modulation depth of GOG is more significant as compared to pure Ge across the whole measurement range from $0.25 \mathrm{THz}$ to $1 \mathrm{THz}$.

For a practical implementation of all-optical terahertz modulators, continuous wave (CW) lasers are more convenient and cost-effective than pulsed femtosecond lasers. Static and dynamic modulation characteristics of the GOG modulator were further investigated at $340 \mathrm{GHz}$ carrier with a CW laser. The detected $\mathrm{THz}$ transmission signals are normalized and plotted in Fig. 3 as a function of the photoexitation power. It can be seen that for both Ge and GOG, the $\mathrm{THz}$ transmission decreases exponentially with the increasing beam power and finally the transmission decay approached a saturation point. At equivalent power levels of the photodoping, GOG has larger attenuation to the $\mathrm{THz}$ wave when compared with Ge. It is shown that for GOG the modulation saturation occurs at a pump power of $0.8 \mathrm{~W}$ and at that power the modulation depth is $94 \%$. At exactly the same pumping power the modulation depth of pure Ge is only $64.2 \%$. This feature of GOG to obtain large modulation depth at low-level photodoing power is very attractive for all-optical $\mathrm{THz}$ devices.

The modulation speed of GOG was measured at $340 \mathrm{GHz}$ carrier. In the measurement, a square-wave voltage was used to drive the $\mathrm{CW}$ diode laser to produce pump beam with the power alternating

(a)

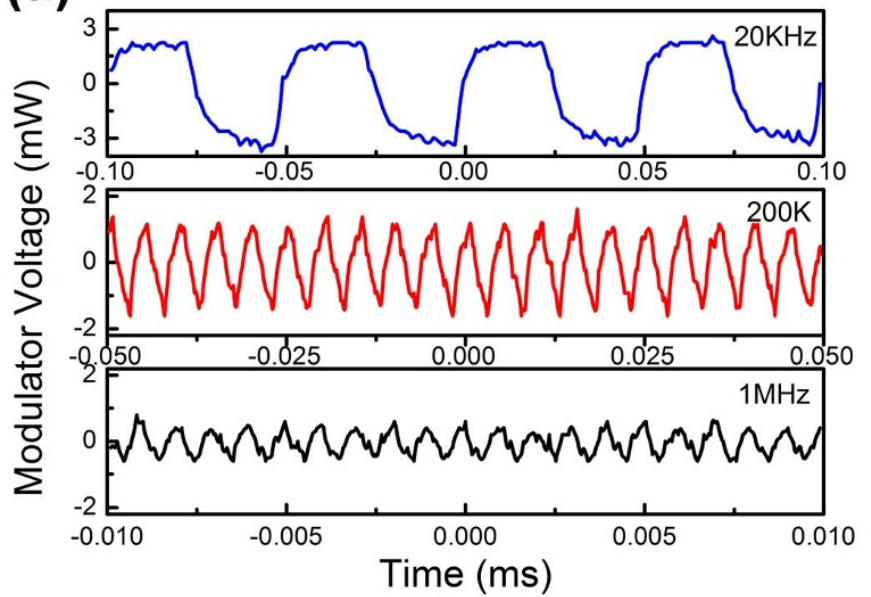

between zero and $200 \mathrm{~mW}$. Here a small laser power of $200 \mathrm{~mW}$ was adopted for the purpose of avoiding the possible accumulation of heating effects. The detected voltage signal is plotted in Fig. 4(a) for different modulation frequencies. Generally, the transmission amplitude of $\mathrm{THz}$ wave decreases with the increase of modulation frequency. At the modulation frequency of $20 \mathrm{KHz}$, the waveform of the modulated $\mathrm{THz}$ signal is almost square, which gradually changes to a triangle shape when the modulation frequencies increase beyond $40 \mathrm{KHz}$. The detected voltage at $100 \mathrm{KHz}$ falls to $78.4 \%$ of that for $20 \mathrm{KHz}$, while at $200 \mathrm{KHz}$ this value notably falls to $\sim 50 \%$. At $1 \mathrm{MHz}$, the device still has a response to the photoexitation but the signal falls to near noise level. The normalized modulation magnitude at different modulation frequency was summarized in Fig. 4(b), which indicate a $3 \mathrm{~dB}$ operation bandwidth $\left(f_{\mathrm{c}}\right)$ is $\sim 200 \mathrm{kHz}$. According to the principle of GOG modulator mentioned above, it is the carrier recombination time of Ge, not graphene, that sets the ultimate limit. Previous researche on germanium crystals show that the carrier lifetimes depend on the resistivity of $\mathrm{Ge}$ and the photo-induced carrier level ${ }^{25}$. Considering the resistivity of Ge used here (about $3 \Omega \cdot \mathrm{cm}$, corresponding to a carrier density of $\sim 10^{6} \mathrm{~cm}^{-3}$ ) and excitation level of $200 \mathrm{~mW}$, the carrier recombination time is in the neighborhood of $2 \mu \mathrm{s}$, suggesting an actual limit of $0.5 \mathrm{MHz}$ for real applications. This result is in good agreement with the directly measured modulation speed of the device. It is reported that a carrier lifetime as short as $30 \mathrm{~ns}$ can be obtained in $\mathrm{Ge}^{25}$. Therefore, the modulation rate of GOG based STM can be tens of megahertz if suitable Ge materials and optical lasers are used.

\section{Discussion}

In a similar structure of graphene on silicon (GOS), a semianalytical model has been proposed to calculate the spectral transmission and the modulation depth of THz radiation ${ }^{20}$. However, by that model the derived numerical results only agree with the experimental data at very low beam power of $30 \mathrm{~mW}$. For higher laser power, the calculated transmissions of GOS deviated qualitatively and quantitatively from the experimental results. The effect of graphene on $\mathrm{THz}$ transmission cannot be well described by that model especially at high modulation intensities. It is reported that graphene is a strong nonlinear material in $\mathrm{THz}$ band and the optical activity of single layer graphene can be significantly enhanced by nonlinear effect ${ }^{26}$. Here we give a qualitative analysis of the observed strong nonlinear transmission in GOG by using the model of nonlinear optical conductivity of monolayer graphene in the terahertz regime $\mathrm{e}^{26,27}$.

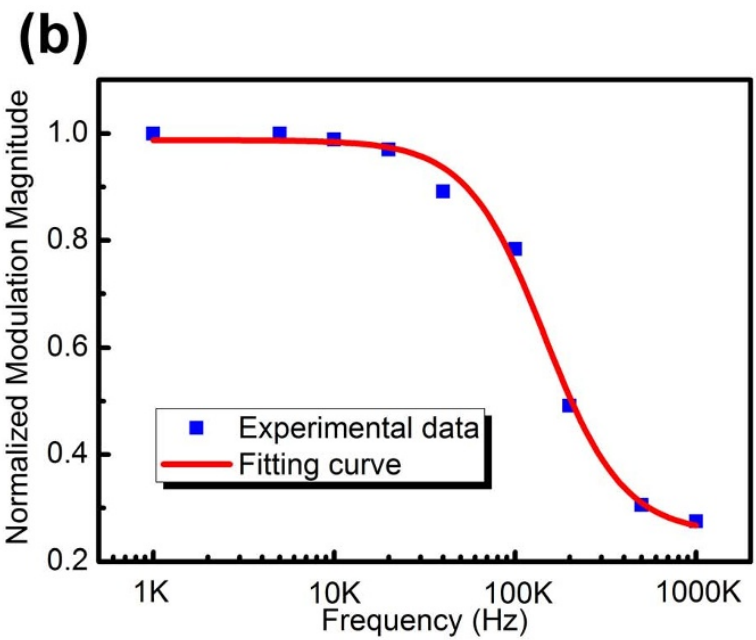

Figure $4 \mid$ Measured dynamic characteristics of the STM. (a) The modulated terahertz beam signal for a carrier frequency of $340 \mathrm{GHz}$ with different modulation frequency. (b) Normalized modulation magnitude, showing a $3 \mathrm{~dB}$ operation bandwidth of $\sim 200 \mathrm{kHz}$. 


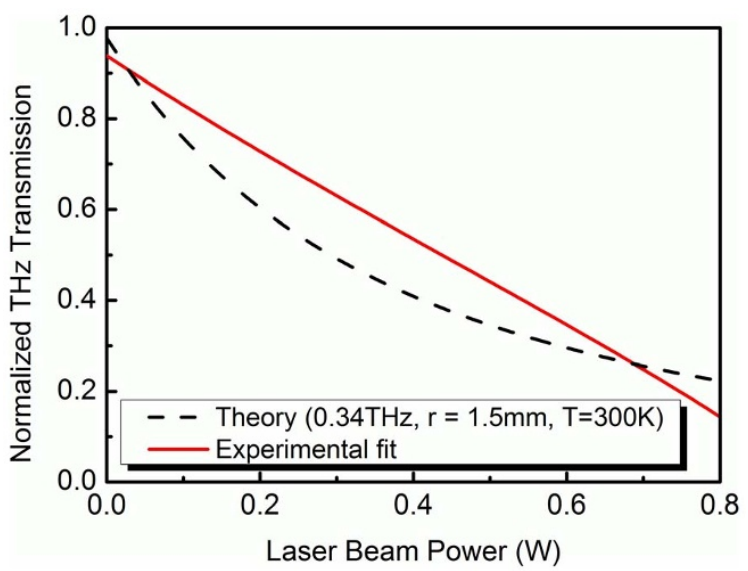

Figure $5 \mid$ Comparison with the model of nonlinear optical response. Normalized transmission coefficient of graphene at $340 \mathrm{GHz}$ carrier as a function of the laser beam power. The black dash line is the theoretical result from Eq.(1) with a radius of the incident laser beam $r=1.5 \mathrm{~mm}$. The beam has been modeled as having a Gaussian distribution. The red solid line is the experimental result of $\mathrm{T}_{\mathrm{SLG}}=\mathrm{T}_{\mathrm{eff}} / \mathrm{T}_{\mathrm{Ge}}$.

The transmission coefficient is given as,

$$
T(E)=\frac{1}{\left(1+\frac{1}{2} \alpha \pi \sigma(E)\right)^{2}}
$$

where $\alpha \approx 1 / 137$ is the fine structure constant. Up to the third order in electric field strength, ignoring the phonon effect and other mechanical effects, the nonlinear conductivity up to the third order nonlinear process can be obtained by using the Floquet expansion ${ }^{26,28}$,

$$
\sigma(E)=\sqrt{\left(1+\sigma_{3}(E) H_{2}\right)^{2}+\sigma_{3}^{2}(E) H_{1}^{2}}
$$

where $\sigma_{3}(E)=\frac{e^{2} v_{F}^{2} E_{0}^{2}}{\hbar^{2} \omega^{4}}, H_{1}=\frac{13}{48} N\left(\frac{1}{2}\right)-\frac{2}{3} N(1)+\frac{45}{48} N\left(\frac{3}{2}\right), H_{2}=$ $2 N(1), N(x)=\tanh \left(\frac{x \hbar \omega}{2 k_{B} T}\right), e$ is the charge of an electron, $v_{F} \approx 1 \times$ $10^{6} \mathrm{~m} / \mathrm{s}$ is the Fermi velocity of graphene, $E_{0}$ is the strength of the incident field, $\omega$ is the frequency of incident photons, and $T$ is the temperature of the system.

The $H_{1}$ and $H_{2}$ terms are a result of taking the time average of the nonlinear currents, $J_{3}(\omega)$ and $J_{3}(3 \omega)$ respectively, in the graphene sheet. The total current is written as the sum of these terms, i.e. $J_{t o t}=$
$J_{1}(\omega)+J_{2}(\omega)+J_{3}(3 \omega)$. When we take the time average of $\left|J_{t o t}\right|^{2}$, the mixed frequency terms, for example $J_{3}(\omega) \cdot J_{3}(3 \omega)$ (and complex conjugate), will disappear, leaving just the un-mixed terms. In terms of pumping power $P$, the third order conductivity of the graphene can be written as,

$$
\sigma_{3}(P)=\frac{e^{2} v_{F}^{2}}{\hbar^{2} \omega^{4}} \frac{2 \ln (2)}{c \varepsilon_{0} \pi r^{2}} P
$$

where $r$ is the approximate radius of the incident laser beam.

For a graphene monolayer on $\mathrm{Ge}$, the effective transmission is the product of the transmission of the monolayer graphene and that of $\mathrm{Ge}, \mathrm{T}_{\text {eff }}=\mathrm{T}_{\mathrm{SLG}} \times \mathrm{T}_{\mathrm{Ge}}$. Therefore the measured $\mathrm{T}_{\mathrm{SLG}}=\mathrm{T}_{\text {eff }} / \mathrm{T}_{\mathrm{Ge}}$. In Figure 5, we compare theoretical result based on Eq.(1) and the measurement at the carrier frequency of $340 \mathrm{GHz}$. In the calculation the diameter of the laser beam is $3 \mathrm{~mm}$ and the system temperature is $300 \mathrm{~K}$. We have modeled the beam as having a Gaussian distribution and have set the FWHM as the beam diameter $(\sim 3 \mathrm{~mm})$. It can be seen the intensity dependence of the transmission coefficient is mainly due to the third order effect in the optical conductivity in terahertz regime. The qualitative agreement is good and the quantitative difference is also quite small even under high laser beam power of $800 \mathrm{~mW}$. The observed quantitative difference may be due to effects neglected in our model such as the imperfectness of the graphene sheet or the inexact profile and size of the laser beam used in the calculation. Works to refine the theoretical model are in progress. Despite the inaccuracy of the model, the theoretical calculations strongly support that the third order effect occurring in graphene result in the enhanced attenuation of $\mathrm{THz}$ transmission in GOG.

In conclusion, we experimentally demonstrated a graphene based all-optical terahertz modulator fabricated on germanium, which can be efficiently driven by a $1.55 \mu \mathrm{m} \mathrm{CW}$ laser at relatively low-level power fluences. We measured a spectrally wide-band modulation of the $\mathrm{THz}$ transmission in the frequency range from 0.25 to $1 \mathrm{THz}$. Dynamic experiments at $340 \mathrm{GHz}$ carrier evidence that GOG provides a $3 \mathrm{~dB}$ modulation speed of $200 \mathrm{KHz}$ and an ultimate limit of $0.5 \mathrm{MHz}$. Theoretical calculations indicates that the laser intensity dependence of the transmission attenuation is mainly due to the third order effect in the optical conductivity of the graphene monolayer. The major advantage of this device is the ability to integrate with low-loss telecommunication fiber thus an all-optical, high speed and low-cost spatial $\mathrm{THz}$ modulator can be readily realized.

\section{Methods}

Sample fabrication and characteristic. The device studied in this work consists of a single-layer of graphene on top of a p-type Ge substrate, as indicated in Fig. 1. The
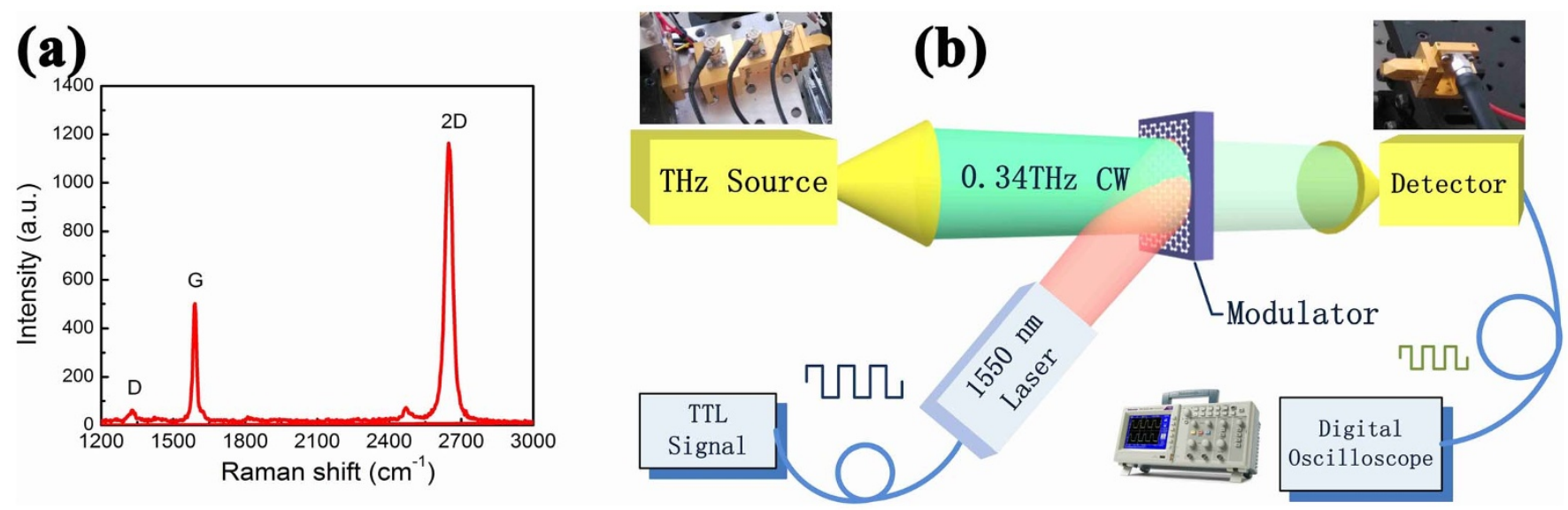

Figure 6 Graphene quality and terahertz characterization set-up. (a) Representative Raman spectrum of the CVD graphene sample. (b) Schematic of the experimental configuration used for Static and dynamic modulation measurements, which using a $340 \mathrm{GHz}$ THz CW source and a $1550 \mathrm{~nm}$ pumping laser. The laser is mounted with a beam incident angle of $30^{\circ}$ to the normal of the modulator. 
modulator was fabricated using chemical vapor deposition (CVD) graphene grown on copper ${ }^{29}$. The Czochralski Ge substrate (Wafer World, Inc) is p-doped with a moderate resistivity of $\sim 3 \Omega \cdot \mathrm{cm}$. The thickness of Ge is $250 \pm 25 \mu \mathrm{m}$. Graphene was transferred by means of polymethyl methacrylate (PMMA) and wet etch methods. Fig. 6(a) is the typical Raman spectrum for our graphene obtained using a $442 \mathrm{~nm}$ excitation laser. The $2 \mathrm{D}$ band is located at $2650 \mathrm{~cm}^{-1}$, and is more than 2 times the height of the corresponding $\mathrm{G}$ band, indicating a single layer graphene on $\mathrm{Ge}$ substrate. Notice that the film we obtained has some defects since a slight $\mathrm{D}$ band was detected.

Terahertz modulator characterization. The measurement set-up for static and dynamic modulation characteristics is shown in Fig. 6(b). The system consists of a VDI (Virginia Diodes) CW terahertz source with a central output in the $340 \mathrm{GHz}$, and a $240-400 \mathrm{GHz}$ zero-bias Schottky diode intensity detector. The average output power of the terahertz source is $\sim 10 \mathrm{~mW}$. A $1.55 \mu \mathrm{m}$ CW diode laser was used to generate the pumping beam with a maximum power of $3 \mathrm{~W}$ and a spot diameter of $\mathrm{d}$ $\sim 3 \mathrm{~mm}(\mathrm{e} . \mathrm{g}, \mathrm{r} \sim 1.5 \mathrm{~mm})$.

1. Chen, H. T. et al. Active terahertz metamaterial devices. Nature 444, 597-600 (2006).

2. Chan, W. L. et al. A spatial light modulator for terahertz beams. Appl. Phys. Lett. 94, 213511 (2009).

3. Kleine-Ostmann, T., Dawson, P., Pierz, K., Hein, G. \& Koch, M. Roomtemperature operation of an electrically driven terahertz modulator. Appl. Phys. Lett. 84, 3555 (2004).

4. Shrekenhamer, D. et al. High speed terahertz modulation from metamaterials with embedded high electron mobility transistors. Opt. Express 19, 9968-9975 (2011).

5. Chen, H. T. et al. Ultrafast Optical Switching of Terahertz Metamaterials Fabricated on ErAs/GaAs Nanoisland Superlattices. Opt. Lett. 32, 1620 (2007)

6. Chen, H. T. et al. Tuning the resonance in high-temperature superconducting terahertz metamaterials. Phys. Rev. Lett. 105, 247402 (2010).

7. Jin, B. B. et al. Low loss and magnetic field-tunable superconducting terahertz metamaterial. Opt. Express 18, 17504-17509 (2010).

8. Wen, Q. Y. et al. Terahertz metamaterials with $\mathrm{VO}_{2}$ cut-wires for thermal tunability. Appl. Phys. Lett. 97, 021111 (2010).

9. Alius, H. \& Dodel, G. Amplitude-, phase-, and frequency modulation of farinfrared radiation by optical excitation of silicon. Infrared Phys. 32, 1 (1991).

10. Vogel, T., Dodel, G., Holzhauer, E., Salzmann, H. \& Theurer, A. High-speed switching of far-infrared radiation by photoionization in a semiconductor. Appl. Opt. 31, 329-337 (1992)

11. Okada, T. \& Tanaka, K. Photo-designed terahertz devices. Sci. Rep. 1, 121; DOI:10.1038/srep00121 (2011)

12. Cheng, L. J. \& Liu, L. Optical modulation of continuous terahertz waves towards cost-effective reconfigurable quasi-optical terahertz components. Opt. Express 21, 28657-28667 (2013).

13. Xie, Z. W. et al. Spatial Terahertz Modulator. Sci. Rep. 3, 3347; DOI:10.1038/ srep03347 (2013).

14. Shrekenhamer, D., Watts, C. M. \& Padilla, W. J. Terahertz single pixer imaging with an optically controlled dynamic spatial light modulator. Opt. Express 21, 12507-12518 (2013)

15. Sensale-Rodriguez, B. et al. Unique prospects for graphene-based terahertz modulators. Appl. Phys. Lett. 99, 113104 (2011).

16. Ju, L. et al. Graphene plasmonics for tunable terahertz metamaterials. Nat Nanotechnol 6, 630-634 (2011).

17. Liu, M. et al. A graphene-based broadband optical modulator. Nature 474, 64-67 (2011).
18. Lee, C. C., Suzuki, S., Xie, W. \& Schibli, T. R. Broadband graphene electro-optic modulators with subwavelength thickness. Opt. Express 20, 5265-5269 (2012).

19. Sensale-Rodriguez, B. et al. Broadband graphene terahertz modulators enabled by intraband transitions. Nat Commun 3, 1-7 (2012).

20. Yao, Y. et al. Broad Electrical Tuning of Graphene-Loaded Plasmonic Antennas. Nano. Lett. 13, 1257-1264 (2013).

21. Li, W. et al. Ultrafast All-Optical Graphene Modulator. Nano. Lett. 14, 955-959 (2014).

22. Weis, P. et al. Spectrally Wide-Band Terahertz Wave Modulator Based on Optically Tuned Graphene. ACS nano, 6, 9118-9124 (2012).

23. Herrscher, M. et al. Epitaxial liftoff InGaAs/InP MSM photodetectors on Si. IEEE Electron. Lett. 31, 1383-1384 (1995).

24. Gatesman, A. J., Waldman, J., Ji, M., Musante, C. \& Yngvesson, S. An AntiReflection Coating for Silicon Optics at Terahertz Frequencies. IEEE Microwave Guided Wave Letters 10, 264-266 (2000).

25. Gaubas, E. \& Vanhellemont, J. Dependence of carrier lifetime in germanium on resisitivity and carrier injection level. Appl. Phys. Lett. 89, 142106 (2006).

26. Wright, A. R., Xu, X. G., Cao, J. C. \& Zhang, C. Strong nonlinear optical response in graphene in terahertz regime. Appl. Phys. Lett. 95, 072101 (2009).

27. Ang, Y. S., Sultan, S. \& Zhang, C. Nonlinear optical spectrum of bilayer graphene in the terahertz regime. Appl. Phys. Lett. 97, 243110 (2010).

28. Zhang, C. Frequency-dependent electrical transport under intense terahertz radiation. Phys. Rev. B 66, (Rapid communication) 081105(R) (2002).

29. Li, X. et al. Large-Area Synthesis of High-Quality and Uniform Graphene Films on Copper Foils. Science 324, 1312-1314 (2009).

\section{Acknowledgments}

This work is supported by National Nature Science Foundation of China (No. 61131005), Keygrant Project of Chinese Ministry of Education (No. 313013), National High-tech Research and Development Projects (No. 2011AA010204), New Century Excellent Talent Foundation (No. NCET-11-0068), and Sichuan Youth S \& T foundation (No. 2011JQ0001)

\section{Author contributions}

Q.Y.W. concerned the devices structure and guided the theoretical and experimental work. W.T. designed and established the $340 \mathrm{GHz}$ measurement system, Q.M. and Z.C. fabricated and characterias the modulator devices. W.W.L. and Q.H.Y. performed the THz-TDS measurements, M.S. developed the theoretical model and did the calculations. H.W.Z. guided the experimental work. All authors discussed the results and co-wrote the manuscript.

\section{Additional information}

Competing financial interests: The authors declare no competing financial interests.

How to cite this article: Wen, Q.-Y. et al. Graphene based All-Optical Spatial Terahertz Modulator. Sci. Rep. 4, 7409; DOI:10.1038/srep07409 (2014)

This work is licensed under a Creative Commons Attribution-NonCommercialShareAlike 4.0 International License. The images or other third party material in this article are included in the article's Creative Commons license, unless indicated otherwise in the credit line; if the material is not included under the Creative Commons license, users will need to obtain permission from the license holder in order to reproduce the material. To view a copy of this license, visit http:// creativecommons.org/licenses/by-nc-sa/4.0/ 